S.-C. Chang

Nagoya Math. J.

Vol. 163 (2001), 193-214

\title{
GLOBAL EXISTENCE AND CONVERGENCE OF SOLUTIONS OF THE CALABI FLOW ON EINSTEIN 4-MANIFOLDS
}

\author{
SHU-CHENG CHANG ${ }^{1}$
}

\begin{abstract}
In this paper, firstly, we show the Bondi-mass type estimate of solutions of Calabi flow on closed 4-manifolds. Secondly, in our applications, we obtain the long time existence on closed 4-manifolds. In particular, we are able to show the asymptotic convergence of a subsequence of solutions of the Calabi flow on closed Einstein 4-manifolds.
\end{abstract}

\section{$\S 1$. Introduction}

Let $\left(M,\left[g_{0}\right]\right)$ be a closed smooth $n$-manifold with a given conformal class $\left[g_{0}\right]$. We consider the scalar curvature functional on $\left[g_{0}\right]$ :

$$
\mathcal{E}(g)=\frac{\int_{M} R^{2} d \mu}{\left(\int_{M} d \mu\right)^{1-4 / n}}, \quad g \in\left[g_{0}\right] .
$$

Then the Euler-Lagrange equation of $\mathcal{E}$ is given by

$$
\Delta R-\beta R^{2}+\beta r=0,
$$

where $d \mu=d \mu_{g}, \Delta=\Delta_{g}, R$ is the scalar curvature with respect to the metric $g, r=\left(\int_{M} R^{2} d \mu\right) /\left(\int_{M} d \mu\right)$ and $\beta=(n-4) / 4(n-1)$.

Now consider the negative gradient flow of $\mathcal{E}$ on a closed smooth $n$ manifold $M$ with a fixed conformal class $\left[g_{0}\right]$ :

$$
\frac{\partial g}{\partial t}=2\left(\Delta R-\beta R^{2}+\beta r\right) g
$$

For $g \in\left[g_{0}\right]$, we may write $g=e^{2 \lambda} g_{0}$, for a smooth function

$$
\lambda: M \times[0, \infty) \longrightarrow \mathbf{R} .
$$

Received October 1, 1999.

1991 Mathematics Subject Classification: Primary 53C21; Secondary 58G03.

${ }^{1}$ Research supported in part by NSC and NCTS Fellow. 
Then equations (1.1) reduce to the following initial value problem of fourth order parabolic equation on $\left(M,\left[g_{0}\right]\right)$ :

$$
\left\{\begin{array}{c}
\frac{\partial \lambda}{\partial t}=\left(\Delta R-\beta R^{2}+\beta r\right) \\
g=e^{2 \lambda} g_{0} ; \lambda(p, 0)=\lambda_{0}(p) \\
\int_{M^{n}} e^{n \lambda_{0}} d \mu_{0}=\int_{M^{n}} d \mu_{0}
\end{array}\right.
$$

where $d \mu_{0}$ is the volume element of $g_{0}$.

In particular, $\beta=0$ for $n=4$, we will consider the following equation on closed 4-manifolds $\left(M^{4},\left[g_{0}\right]\right)$ :

$$
\left\{\begin{array}{l}
\frac{\partial \lambda}{\partial t}=\Delta R \\
g=e^{2 \lambda} g_{0} ; \lambda(p, 0)=\lambda_{0}(p) \\
\int_{M^{4}} e^{4 \lambda_{0}} d \mu_{0}=\int_{M^{4}} d \mu_{0}
\end{array}\right.
$$

The parabolic equation (1.3) is so-called Calabi flow in case of Kaehler surfaces with the fixed Kaehler class due to E. Calabi ([Ca], [Ch2], [Ch3]).

For $n=2$ and 3, based on the Bondi-mass type estimate of solutions of (1.2) and [Chru], the present author proved the long time existence and asymptotic convergence of solutions of (1.2). We refer to [CW] and [Ch5] for details.

In this paper, firstly, we show the Bondi-mass type estimate of solutions of the Calabi flow (1.3) as in Corollary 2.3. Secondly, based on Corollary 2.3 and elliptic Moser iteration plus blowing-up argument as in [Ch1], we have the $C^{0}$-bound and $W_{k, 2}$-norms bounds as in Theorem 3.6 and Theorem 3.7. Then the long time existence of solutions of (1.3) was claimed. Finally, we show the asymptotic convergence of solutions of (1.3) if the background metric $g_{0}$ is Einstein.

Let $Q$ be the Yamabe constant on $\left(M^{4},\left[g_{0}\right]\right)$ which is conformal invariant

$$
Q\left(M, g_{0}\right)=\inf _{\varphi \neq 0} \frac{E_{g_{0}}(\varphi)}{\left(\int|\varphi|^{4} d \mu_{0}\right)^{1 / 2}},
$$

where $E_{g_{0}}(\varphi)=\int\left|\frac{0}{\nabla} \varphi\right|^{2} d \mu_{0}+\frac{1}{6} \int R_{0} \varphi^{2} d \mu_{0}$. 
THEOREM 1.1. Let $\left(M, g_{0}\right)$ be a closed 4-manifold and $\lambda$ satisfy (1.3) on $[0, T)$. Then the solution of $(1.3)$ exists on $M \times[0, \infty)$. Moreover, if $g_{0}$ is an Einstein metric, there exists a subsequence of solutions $\left\{e^{2 \lambda(t)} g_{0}\right\}$ of (1.3) on $M \times[0, \infty)$ which converges smoothly to one of the constant scalar curvature metric $g_{\infty}$.

Remark 1.1. In case of Kaehler surfaces with the fixed Kaehler class, we have the similar results as in Theorem 1.1 with the stability condition on the tangent bundle ([Ch2]).

One may think the problem here to be more difficult compare the second order parabolic equations, due to a lack of the maximum principle for fourth order parabolic equations. Then in order to estimate the $C^{0}$-bound, we will apply the elliptic Moser iteration method plus the blow-up argument ([Ch1]).

In Section 2, we will derive the so-called Bondi-mass type estimate of equation (1.3) from the Bochner formula. In Section 3, based on [Ch1], [CY], we obtain the $C^{0}$-bound via elliptic Moser iteration and the blow-up argument. Then the higher order $W_{k, 2}$-norms estimates of the solutions for (1.3) will follow easily from [CW] and [Chru]. Finally, we have the long-time existence of solutions of (1.3).

In Section 4, we are able to show the asymptotic convergence of a subsequence of solutions of (1.3) if the background metric $g_{0}$ is Einstein.

Acknowledgements. I would like to express my thanks to Prof. S.T. Yau for constant encouragement and for the hospitality during his visit at Harvard University.

\section{$\S 2$. Bondi-mass type estimates of solutions of the Calabi flow}

In this section, we will derive the key estimate of equation (1.3) from the Bochner formula as in Lemma 2.2. This is so-called the Bondi-mass type estimate as in [Ch5] and [CW].

For $g=e^{2 \lambda} g_{0}, R_{0}=R_{g_{0}}$, we have the following formulae for (1.3):

$$
\begin{gathered}
R=R_{g}=e^{-2 \lambda}\left(R_{0}-6 \Delta_{0} \lambda-6|\stackrel{0}{\nabla} \lambda|^{2}\right) . \\
\Delta R=e^{-2 \lambda}\left(\Delta_{0} R+2\langle\stackrel{0}{\nabla} R, \stackrel{0}{\nabla} \lambda\rangle\right), \quad \text { where } \Delta_{0}=\Delta_{g_{0}}, \Delta=\Delta_{g} . \\
d \mu=e^{4 \lambda} d \mu_{0}, \quad \text { where } d \mu_{0}=d \mu_{g_{0}}, d \mu=d \mu_{g} .
\end{gathered}
$$




$$
\begin{gathered}
\frac{\partial}{\partial t} d \mu=4 \Delta R d \mu ; \quad \frac{\partial R}{\partial t}=-2 R \Delta R-6 \Delta^{2} R \\
\int_{M^{4}} d \mu=\int_{M} e^{4 \lambda} d \mu_{0}=\int_{M} e^{4 \lambda_{0}} d \mu_{0}=\int_{M} d \mu_{0} .
\end{gathered}
$$

LEMMA 2.1. Under the flow (1.3), we have

$$
\int_{M} R^{2} d \mu \leq C\left(R_{0}, \lambda_{0}\right),
$$

for $0 \leq T \leq \infty$

Proof. From (2.4),

$$
\begin{aligned}
-\frac{1}{2} \frac{d}{d t} \int_{M} R^{2} d \mu & =-2 \int_{M} R^{2} \Delta R d \mu+2 \int\left(R^{2} \Delta R+3 R \Delta^{2} R\right) d \mu \\
& =6 \int_{M}(\Delta R)^{2} d \mu .
\end{aligned}
$$

Thus

$$
\frac{d}{d t} \int_{M} R^{2} d \mu \leq 0 .
$$

Compare with [CW, Theorem 2.4] and [Ch5], one can show

LEMMA 2.2. (i) ([Ch4]) For $g_{0}$ is Einstein, under the flow (1.3), we have

$$
\frac{d}{d t} \int_{M} e^{5 \lambda} d \mu_{0} \leq 0
$$

(ii) For any background metric $g_{0}$, under the flow (1.3), we have

$$
\frac{d}{d t} \int_{M} e^{5 \lambda} d \mu_{0} \leq C\left(g_{0}, \lambda_{0}\right)
$$

Remark 2.1. (i) We will need (ii) for long time existence part and (i) for convergence part of the Calabi flow.

(ii) The volume $\int_{M} d \mu=\int_{M} e^{4 \lambda} d \mu_{0}$ will be preserved under the flow. 
Proof. In the following, the constant $C$ may vary from line to line which is independent of $t$. From (2.1) and (2.2), we have

$$
\begin{aligned}
\frac{d}{d t} \int_{M} e^{\alpha \lambda} d \mu_{0} & =\alpha \int e^{\alpha \lambda}\left(\frac{\partial \lambda}{\partial t}\right) d \mu_{0}=\alpha \int e^{\alpha \lambda}(\Delta R) d \mu_{0} \\
= & \alpha \int e^{\alpha \lambda} \cdot e^{-2 \lambda}\left(\Delta_{0} R+2\langle\stackrel{0}{\nabla} R, \stackrel{0}{\nabla} \lambda\rangle\right) d \mu_{0} \\
= & \alpha \int e^{(\alpha-2) \lambda} R\left[(\alpha-4) \Delta_{0} \lambda+\left.(\alpha-2)(\alpha-4)||^{0} \lambda\right|^{2}\right] d \mu_{0} \\
= & \alpha \int e^{(\alpha-4) \lambda}\left[(\alpha-4) R_{0} \Delta_{0} \lambda+\left.(\alpha-2)(\alpha-4) R_{0}||^{0} \nabla\right|^{2}\right. \\
& \quad-6(\alpha-4)\left(\Delta_{0} \lambda\right)^{2}-\left.3(\alpha-4)(2 \alpha-2) \Delta_{0} \lambda||^{0} \nabla \lambda\right|^{2} \\
& \left.\quad-\left.\left.6(\alpha-2)(\alpha-4)\right|^{0} \lambda\right|^{4}\right] d \mu_{0} .
\end{aligned}
$$

Now let $f=e^{(4-\alpha) \lambda}$. Then

$$
\begin{aligned}
|\stackrel{0}{\nabla} \lambda|^{2} & =(\alpha-4)^{-2} f^{-2}|\stackrel{0}{\nabla} f|^{2}, \\
\Delta_{0} \lambda & =(\alpha-4)^{-1} f^{-2}|\stackrel{0}{\nabla} f|^{2}-(\alpha-4)^{-1} f^{-1} \Delta_{0} f .
\end{aligned}
$$

Hence

$$
\begin{aligned}
\frac{d}{d t} \int e^{\alpha \lambda} d \mu_{0}=2 \alpha & (\alpha-4) \int e^{(\alpha-4) \lambda} R_{0}|\stackrel{0}{\nabla} \lambda|^{2} d \mu_{0} \\
& -\alpha(\alpha-4) \int e^{(\alpha-4) \lambda}\left\langle\stackrel{0}{\nabla} \lambda, \stackrel{0}{\nabla} R_{0}\right\rangle d \mu_{0} \\
& -6 \alpha(\alpha-4)^{-1} \int f^{-3}\left(\Delta_{0} f\right)^{2} d \mu_{0} \\
& -3 \alpha(\alpha-4)^{-3}\left(4 \alpha^{2}-24 \alpha+36\right) \int f^{-5}|\stackrel{0}{\nabla} f|^{4} d \mu_{0} \\
& +\left.\left.3 \alpha(\alpha-4)^{-2}(6 \alpha-18) \int f^{-4} \Delta_{0} f\right|^{0} \nabla\right|^{2} d \mu_{0} .
\end{aligned}
$$

Again let $F=f^{r}$, for some $r$ to be chosen later. Then

$$
\begin{aligned}
|\stackrel{0}{\nabla} f|^{2} & =r^{-2} F^{(2-2 r) / r}|\stackrel{0}{\nabla} F|^{2}, \\
\Delta_{0} f & =r^{-1} F^{(1-r) / r} \Delta_{0} F-(r-1) r^{-2} F^{(1-2 r) / r}\left|{ }^{0} \nabla F\right|^{2} .
\end{aligned}
$$


Then

$$
\begin{aligned}
& \frac{d}{d t} \int e^{\alpha \lambda} d \mu_{0}=2 \alpha(\alpha-4) \int e^{(\alpha-4) \lambda} R_{0}|\stackrel{0}{\nabla} \lambda|^{2} d \mu_{0} \\
& \quad-\alpha(\alpha-4) \int e^{(\alpha-4) \lambda}\left\langle\stackrel{0}{\nabla} \lambda, \stackrel{0}{\nabla} R_{0}\right\rangle d \mu_{0} \\
& \quad-6 \alpha(\alpha-n)(\alpha-4)^{-1} r^{-2} \int F^{(-1-2 r) / r}\left(\Delta_{0} F\right)^{2} d \mu_{0} \\
& \quad-6 \alpha(\alpha-4)^{-3} r^{-4}\left[\left(r(r+1) \alpha^{2}-\left(8 r^{2}+5 r-1\right) \alpha\right.\right. \\
& \left.\quad+16 r^{2}+4 r-2\right] \int F^{(-1-4 r) / r}|\stackrel{0}{\nabla} F|^{4} d \mu_{0} \\
& +\left.\left.6 \alpha(\alpha-4)^{-2} r^{-3}[(2 r+1) \alpha-8 r-1] \int F^{(-1-3 r) / r} \Delta_{0} F\right|^{0} \nabla F\right|^{2} d \mu_{0}
\end{aligned}
$$

Compute

$$
\begin{aligned}
0= & \int_{M} \delta\left(F^{-\frac{1}{r}-2} \stackrel{0}{\nabla} F \Delta_{0} F\right) d \mu_{0} \\
= & \int F^{-\frac{1}{r}-2}\left(\Delta_{0} F\right)^{2} d \mu_{0}+\int\left\langle\stackrel{0}{\nabla}\left(F^{-\frac{1}{r}-2} \Delta_{0} F\right), \stackrel{0}{\nabla} F\right\rangle d \mu_{0} \\
= & \int F^{-\frac{1}{r}-2}\left(\Delta_{0} F\right)^{2} d \mu_{0}-\left(\frac{1}{r}+2\right) \int F^{-\frac{1}{r}-3} \Delta_{0} F|\stackrel{0}{\nabla} F|^{2} d \mu_{0} \\
& \quad+\int F^{-\frac{1}{r}-2}\left\langle\stackrel{0}{\nabla} \Delta_{0} F, \stackrel{0}{\nabla} F\right\rangle d \mu_{0},
\end{aligned}
$$

and

$$
\begin{aligned}
0= & \int_{M} \delta\left(F^{-\frac{1}{r}-2} \stackrel{0}{\nabla}|\stackrel{0}{\nabla} F|^{2}\right) d \mu_{0} \\
= & \int F^{-\frac{1}{r}-2} \Delta_{0}|\stackrel{0}{\nabla} F|^{2} d \mu_{0}-\left(\frac{1}{r}+2\right) \int F^{-\frac{1}{r}-3}\left\langle\stackrel{0}{\nabla} F,\left.\left.\stackrel{0}{\nabla}\right|^{0} \nabla F\right|^{2} d \mu_{0}\right\rangle d \mu_{0} \\
= & \int F^{-\frac{1}{r}-2} \Delta_{0}|\stackrel{0}{\nabla} F|^{2} d \mu_{0}+\left.\left.\left(\frac{1}{r}+2\right) \int F^{-\frac{1}{r}-3} \Delta_{0} F\right|^{0} \nabla F\right|^{2} d \mu_{0} \\
& \quad-\left(\frac{1}{r}+2\right)\left(\frac{1}{r}+3\right) \int F^{-\frac{1}{r}-4}|\stackrel{0}{\nabla} F|^{4} d \mu_{0} .
\end{aligned}
$$

By the Bochner-Lichnerowicz formula

$$
\frac{1}{2} \Delta_{0}|\stackrel{0}{\nabla} F|^{2}=\left|\stackrel{0}{\nabla}^{2} F\right|^{2}+\left\langle\stackrel{0}{\nabla}^{2} F, \stackrel{0}{\nabla} \Delta_{0} F\right\rangle+R c(\stackrel{0}{\nabla} F, \stackrel{0}{\nabla} F)
$$


we have

$$
\begin{aligned}
\int F^{-\frac{1}{r}-2}\left\langle\stackrel{0}{\nabla} F, \stackrel{0}{\nabla} \Delta_{0} F\right\rangle d \mu_{0} \\
=-\int F^{-\frac{1}{r}-2}\left|\stackrel{0}{\nabla^{2}} F\right|^{2} d \mu_{0}-\int F^{-\frac{1}{r}-2} R c(\stackrel{0}{\nabla} F, \stackrel{0}{\nabla} F) d \mu_{0} \\
\quad-\frac{1}{2}\left(\frac{1}{r}+2\right) \int F^{-\frac{1}{r}-3} \Delta_{0} F|\stackrel{0}{\nabla} F|^{2} d \mu_{0} \\
\quad+\frac{1}{2}\left(\frac{1}{r}+2\right)\left(\frac{1}{r}+3\right) \int F^{-\frac{1}{r}-4}|\stackrel{0}{\nabla} F|^{4} d \mu_{0} .
\end{aligned}
$$

Combine (2.6) and (2.7), one obtains

$$
\begin{aligned}
& \frac{3}{2}\left(\frac{1}{r}+2\right) \int F^{-\frac{1}{r}-3} \Delta_{0} F|\stackrel{0}{\nabla} F|^{2} d \mu_{0} \\
& =\int F^{-\frac{1}{r}-2}\left(\Delta_{0} F\right)^{2} d \mu_{0} \\
& \quad+\frac{1}{2}\left(\frac{1}{r}+2\right)\left(\frac{1}{r}+3\right) \int F^{-\frac{1}{r}-4}|\stackrel{0}{\nabla} F|^{4} d \mu_{0} \\
& \quad-\int F^{-\frac{1}{r}-2}\left|\nabla^{2} F\right|^{2} d \mu_{0}-\int F^{-\frac{1}{r}-2} R c(\stackrel{0}{\nabla} F, \stackrel{0}{\nabla} F) d \mu_{0} .
\end{aligned}
$$

Hence

$$
\begin{aligned}
& \int F^{-\frac{1}{r}-3} \Delta_{0} F|\stackrel{0}{\nabla} F|^{2} d \mu_{0} \\
&=\frac{2}{3} \frac{r}{1+2 r} \int F^{-\frac{1}{r}-2}\left(\Delta_{0} F\right)^{2} d \mu_{0}-\frac{2}{3} \frac{r}{1+2 r} \int F^{-\frac{1}{r}-2}\left|\nabla^{2} F\right|^{2} d \mu_{0} \\
&-\frac{2}{3} \frac{r}{1+2 r} \int F^{-\frac{1}{r}-2} R c(\stackrel{0}{\nabla} F, \stackrel{0}{\nabla} F) d \mu_{0} \\
&+\frac{1}{3} \frac{1+3 r}{r} \int F^{-\frac{1}{r}-4}|\stackrel{0}{\nabla} F|^{4} d \mu_{0} .
\end{aligned}
$$

Then

$$
\begin{aligned}
& \frac{d}{d t} \int e^{\alpha \lambda} d \mu_{0}=2 \alpha(\alpha-4) \int e^{(\alpha-4) \lambda} R_{0}\left|\nabla^{0} \lambda\right|^{2} d \mu_{0} \\
& \quad-\alpha(\alpha-4) \int e^{(\alpha-4) \lambda}\left\langle\stackrel{0}{\nabla} \lambda,{\left.\stackrel{0}{\nabla} R_{0}\right\rangle d \mu_{0}}^{-2} r^{-2}\left[(\alpha-4)-6(2 r+1)^{-1}\right] \int F^{-\frac{1}{r}-2}\left(\Delta_{0} F\right)^{2} d \mu_{0}\right.
\end{aligned}
$$




$$
\begin{aligned}
& +2 \alpha(\alpha-4)^{-3} r^{-4}\left[\left(3 r^{2}+2 r+1\right)(\alpha-4)^{2}-6\right] \int F^{-\frac{1}{r}-4}\left|\nabla^{0} F\right|^{4} d \mu_{0} \\
& -4 \alpha(\alpha-4)^{-2} r^{-2}\left[\alpha-(8 r+1)(1+2 r)^{-1}\right] \int F^{-\frac{1}{r}-2}\left|\nabla^{2} F\right|^{2} d \mu_{0} \\
& -4 \alpha(\alpha-4)^{-2} r^{-2}\left[\alpha-(8 r+1)(1+2 r)^{-1}\right] \int F^{-\frac{1}{r}-2} R c(\stackrel{0}{\nabla} F, \stackrel{0}{\nabla} F) d \mu_{0} .
\end{aligned}
$$

(i) For $g_{0}$ is Einstein, i.e., $R c\left(g_{0}\right)=\left(R_{0} / 4\right) g_{0}$ :

Choose $r=1, \alpha=5$, then

$$
\begin{aligned}
\frac{d}{d t} \int e^{5 \lambda} d \mu_{0}= & 10 R_{0} \int e^{\lambda}|\stackrel{0}{\nabla} \lambda|^{2} d \mu_{0}-40 \int e^{3 \lambda} R c(\stackrel{0}{\nabla} F, \stackrel{0}{\nabla} F) d \mu_{0} \\
& +10 \int e^{3 \lambda}\left(\Delta_{0} F\right)^{2} d \mu_{0}-40 \int e^{3 \lambda}\left|\stackrel{0}{\nabla}^{2} F\right|^{2} d \mu_{0} \\
= & 10 \int e^{3 \lambda}\left(\Delta_{0} F\right)^{2} d \mu_{0}-40 \int e^{3 \lambda}\left|\nabla^{2} F\right|^{2} d \mu_{0}
\end{aligned}
$$

But for $n=4$,

$$
\left(\Delta_{0} F\right)^{2} \leq 4\left|\nabla^{0} F\right|^{2} .
$$

This implies (i) of the Lemma.

(ii) For any arbitrary $g_{0}$ :

First we observe, for $0 \leq \beta \leq 4 ; \Omega^{+}=\{p \in M \mid \lambda \geq 0\}, \Omega^{-}=\{p \in$ $M \mid \lambda<0\}$

$$
\begin{aligned}
\int_{M} e^{\beta \lambda} d \mu_{0} & =\int_{\Omega^{+}} e^{\beta \lambda} d \mu_{0}+\int_{\Omega^{-}} e^{\beta \lambda} d \mu_{0} \leq \int_{M} e^{4 \lambda} d \mu_{0}+\int_{M} d \mu_{0} \\
& \leq \int_{M} d \mu+\int_{M} d \mu_{0} \leq C
\end{aligned}
$$

Choose $r=1, \alpha=5$ again, then, from (2.8) and (2.9),

$$
\begin{gathered}
\frac{d}{d t} \int e^{5 \lambda} d \mu_{0} \leq 10 \int e^{\lambda}|\stackrel{0}{\nabla} \lambda|^{2} R_{0} d \mu_{0}-40 \int e^{\lambda} R c(\stackrel{0}{\nabla} \lambda, \stackrel{0}{\nabla} \lambda) d \mu_{0} \\
-5 \int e^{\lambda}\left\langle\stackrel{0}{\nabla} \lambda, \stackrel{0}{\nabla} R_{0}\right\rangle d \mu_{0}
\end{gathered}
$$

Compute, for $e^{2 \lambda} R=R_{0}-6 \Delta_{0} \lambda-6\left|{ }^{0} \nabla \lambda\right|^{2}$, from (2.5), (2.10)

$$
-5 \int e^{\lambda}\left\langle\stackrel{0}{\nabla} \lambda, \stackrel{0}{\nabla} R_{0}\right\rangle d \mu_{0}=5 \int e^{\lambda}|\stackrel{0}{\nabla} \lambda|^{2} R_{0} d \mu_{0}+5 \int e^{\lambda}\left(\Delta_{0} \lambda\right) R_{0} d \mu_{0}
$$




$$
\begin{aligned}
& =\frac{5}{6} \int e^{\lambda}\left(-e^{2 \lambda} R+R_{0}\right) R_{0} d \mu_{0} \\
& =\frac{5}{6} \int e^{\lambda} R_{0}^{2} d \mu_{0}-\frac{5}{6} \int e^{3 \lambda} R R_{0} d \mu_{0} \\
& \leq C \int e^{\lambda} d \mu_{0}+C \int e^{2 \lambda} d \mu_{0}+C \int e^{4 \lambda} R^{2} d \mu_{0} \\
& \leq C
\end{aligned}
$$

and

$$
\begin{aligned}
& 10 \int_{M} e^{\lambda}|\stackrel{0}{\nabla} \lambda|^{2} R_{0} d \mu_{0}-40 \int_{M} e^{\lambda} R c(\stackrel{0}{\nabla} \lambda, \stackrel{0}{\nabla} \lambda) d \mu_{0} \\
& \leq C \int_{M} e^{\lambda}|\stackrel{0}{\nabla} \lambda|^{2} d \mu_{0} \\
& \quad \leq C \int_{\Omega^{+}} e^{2 \lambda}|\stackrel{0}{\nabla} \lambda|^{2} d \mu_{0}+C \int_{\Omega^{-}} e^{\lambda / 2}|\stackrel{0}{\nabla} \lambda|^{2} d \mu_{0} \\
& \leq C \int_{M} e^{2 \lambda}|\stackrel{0}{\nabla} \lambda|^{2} d \mu_{0}+C \int_{M} e^{\lambda / 2}|\stackrel{0}{\nabla} \lambda|^{2} d \mu_{0} .
\end{aligned}
$$

Now

$$
3 \Delta_{0} e^{2 \lambda}=e^{2 \lambda}\left(6 \Delta_{0} \lambda+12|\stackrel{0}{\nabla} \lambda|^{2}\right)=e^{2 \lambda}\left(-e^{2 \lambda} R+R_{0}+6|\stackrel{0}{\nabla} \lambda|^{2}\right)
$$

and

$$
12 \Delta_{0} e^{\lambda / 2}=e^{\lambda / 2}\left(6 \Delta_{0} \lambda+3|\stackrel{0}{\nabla} \lambda|^{2}\right)=e^{\lambda / 2}\left(-e^{2 \lambda} R+R_{0}-3|\stackrel{0}{\nabla} \lambda|^{2}\right) .
$$

It follows

$$
\begin{aligned}
6 \int e^{2 \lambda}|\stackrel{0}{\nabla} \lambda|^{2} d \mu_{0} & =-\int e^{2 \lambda} R_{0} d \mu_{0}+\int e^{4 \lambda} R d \mu_{0} \\
& =-\int e^{2 \lambda} R_{0} d \mu_{0}+\int R d \mu \leq C
\end{aligned}
$$

and

$$
\begin{aligned}
3 \int e^{\lambda / 2}\left|\nabla^{0} \lambda\right|^{2} d \mu_{0} & =\int e^{\lambda / 2} R_{0} d \mu_{0}-\int e^{5 \lambda / 2} R d \mu_{0} \\
& \leq C-\int e^{-3 \lambda / 2} R d \mu \\
& \leq C+\int e^{-3 \lambda} d \mu+\int R^{2} d \mu \\
& \leq C+\int e^{\lambda} d \mu_{0}+\int R^{2} d \mu \leq C
\end{aligned}
$$

Then (2.11), (2.12) and (2.13) imply (ii) of the Lemma. 
Corollary 2.3. (i) For $g_{0}$ is Einstein, under the flow (1.3), one obtains

$$
\int_{M} e^{5 \lambda} d \mu_{0} \leq C_{1}\left(g_{0}, \lambda_{0}\right)
$$

for all $0 \leq t \leq T \leq \infty$.

(ii) For any background metric $g_{0}$, then

$$
\int_{M} e^{5 \lambda} d \mu_{0} \leq C_{2}\left(g_{0}, \lambda_{0}\right)+C_{3}\left(g_{0}, \lambda_{0}\right) t,
$$

for all $0 \leq t<T$.

Remark 2.2. From (ii) of Corollary 2.3, under the flow (1.3), we have

$$
\int_{M} e^{5 \lambda} d \mu_{0} \leq C_{2}+C_{3} t
$$

This will be enough for the long time existence of the solution of (1.3) which will imply the first part of assertion for Theorem 1.1. But for convergence part, we will need the uniformly bound on $\int_{M} e^{5 \lambda} d \mu_{0}$ under the flow (1.3) which is held when $g_{0}$ is Einstein as in (i) of Corollary 2.3.

\section{$\S 3$. A priori estimates and long time existence}

In this section, following Corollary 2.3, [Ch1] and [CY], we will have the $C^{0}$-bound via elliptic Moser iteration and the blow-up argument as in Theorem 3.6. Then, based on [CW] and [Chru], one can get the bounds on all $W_{k, 2}$ norms as in Theorem 3.7. All these together will imply the long-time existence of solutions of (1.3).

Define

$$
E_{\eta}=\left\{p: e^{\lambda(p)} \geq \eta\right\}, \quad\left|E_{\eta}\right|=\int_{E_{\eta}} d \mu_{0} .
$$

In the following, the constant $C$ may vary from line to line.

LEMMA 3.1. Under the flow (1.3), there exists $\eta_{0}>0, l_{0}>0$ such that

$$
\left|E_{\eta_{0}}\right| \geq l_{0}>0
$$

for all $0 \leq t<T$. 
Proof. First, from the Corollary 2.3 (we refer to Remark 3.1 as below), we may choose $0<\epsilon \leq 1$ such that

$$
\int_{M} e^{(4+\epsilon) \lambda} d \mu_{0} \leq C
$$

Now

$$
\begin{aligned}
\left(\int_{M} e^{4 \lambda} d \mu_{0}\right)^{2} & \leq\left(\int_{M} e^{(4-\epsilon) \lambda} d \mu_{0}\right)\left(\int_{M} e^{(4+\epsilon) \lambda} d \mu_{0}\right) \\
& \leq C \int_{M} e^{(4-\epsilon) \lambda} d \mu_{0}
\end{aligned}
$$

Since $\int_{M} e^{4 \lambda} d \mu_{0}$ is fixed under the flow (1.3), say $\int_{M} e^{4 \lambda} d \mu_{0}=V$. Thus

$$
\frac{V^{2}}{C} \leq \int_{M} e^{(4-\epsilon) \lambda} d \mu_{0}
$$

But, for all $\eta>0$,

$$
\begin{aligned}
\int_{M} e^{(4-\epsilon) \lambda} d \mu_{0} & =\int_{E_{\eta}} e^{(4-\epsilon) \lambda} d \mu_{0}+\int_{E_{\eta}^{c}} e^{(4-\epsilon) \lambda} d \mu_{0} \\
& \leq\left(\int_{E_{\eta}} e^{4 \lambda} d \mu_{0}\right)^{(4-\epsilon) / 4}\left(E_{\eta}\right)^{\epsilon / 4}+\eta^{(4-\epsilon)}\left|E_{\eta}^{c}\right|
\end{aligned}
$$

Now for sufficiently small $\eta_{0}$, say

$$
\eta_{0}^{4-\epsilon} V<\frac{1}{2} \frac{V^{2}}{C}
$$

Then

$$
\frac{1}{2} \frac{V^{2}}{C} \leq(V)^{(4-\epsilon) / 4}\left(E_{\eta_{0}}\right)^{\epsilon / 4} .
$$

This implies, for $l_{0}=V(V / 2 C)^{4 / \epsilon}$

$$
\left|E_{\eta_{0}}\right| \geq l_{0}
$$

Remark 3.1. The delicate part of the proof in $[\mathrm{CY}]$ is the estimate (3.1) which is held for $\epsilon=1$ under the flow (1.3) due to Lemma 2.2. If $g_{0}$ is Einstein, then $l_{0}$ is independent of the maximum time $T \leq \infty$. If $g_{0}$ is not Einstein, then the estimate is still held as long as the maximum time $T$ is finite. 
LEMMA 3.2. Under the flow (1.3), there exists a constant $C>0$ such that

$$
\int_{M} \lambda^{2} d \mu_{0} \leq C
$$

for $0 \leq t<T$.

Proof. Choose $\eta_{0}, l_{0}$ as in Lemma 3.1, for $D=E_{\eta_{0}}^{c}$, consider the Raleigh-Ritz characterization for $\lambda_{1}(D)$, one has

$$
\int_{D}\left|\ln \frac{e^{\lambda}}{\eta_{0}}\right|^{2} d \mu_{0} \leq \frac{1}{\lambda_{1}(D)} \int_{D}\left|\stackrel{0}{\nabla}^{\prime} \ln \frac{e^{\lambda}}{\eta_{0}}\right|^{2} d \mu_{0} .
$$

From Lemma 3.1, we have

$$
|D|=V-\left|E_{\eta_{0}}\right| \leq V-l_{0}
$$

Then, from Faber-Krahn inequality ([Chav])

$$
\lambda_{1}(D) \geq C\left(l_{0}\right)>0 \text {. }
$$

Now

$$
\begin{aligned}
\int_{e^{\lambda} \leq \eta_{0}}\left|\ln \frac{e^{\lambda}}{\eta_{0}}\right|^{2} d \mu_{0} & \leq \frac{1}{C\left(l_{0}\right)} \int_{M}\left|\frac{0}{\nabla} \ln \frac{e^{\lambda}}{\eta_{0}}\right|^{2} d \mu_{0} \\
& =\frac{1}{C\left(l_{0}\right)} \int_{M} \frac{\Delta_{0} e^{\lambda}}{e^{\lambda}} d \mu_{0} \\
& =\frac{1}{C\left(l_{0}\right)} \int_{M}\left(\Delta_{0} \lambda+\left|\nabla^{0} \lambda\right|^{2}\right) d \mu_{0} \\
& =\frac{1}{C\left(l_{0}\right)} \int_{M}\left(R_{0}-e^{2 \lambda} R\right) d \mu_{0} \\
& \leq \frac{1}{C\left(l_{0}\right)}\left(\int_{M} R_{0} d \mu_{0}+\frac{1}{2} \int_{M} R^{2} d \mu+\frac{1}{2} \int_{M} d \mu\right) \\
& \leq C .
\end{aligned}
$$

On the other hand

$$
\int_{e^{\lambda} \geq \eta_{0}}\left|\ln \frac{e^{\lambda}}{\eta_{0}}\right|^{2} d \mu_{0} \leq \int_{e^{\lambda} \geq \eta_{0}}\left|\frac{e^{\lambda}}{\eta_{0}}\right|^{2} d \mu_{0} \leq \frac{1}{\eta_{0}^{2}} \int_{M} e^{2 \lambda} d \mu_{0} \leq C .
$$

All these imply the Lemma. 
Now, by using the result of Lemma 3.2, we have the local Sobolev constant bound $C_{s}$ with respect to $g$.

LEMMA 3.3. Under the flow (1.3), there exists a constant $\kappa_{1}>0$ such that, if

$$
\int_{B_{\rho}} R^{2} d \mu \leq \kappa_{1}
$$

then, for some constant $C_{s}$

$$
\left(\int_{B_{\rho}} f^{4} d \mu\right)^{1 / 2} \leq C_{s}\left[\int_{B_{\rho}}|\nabla f|^{2} d \mu+\int_{B_{\rho}} f^{2} d \mu\right]
$$

for $f \in C_{0}^{\infty}\left(B_{\rho}\right)$.

Proof. For $g=e^{2 \lambda} g_{0}$ and $n=4$ in our case. Now with respect to $g_{0}$, we have the local Sobolev constant $A_{0}$, i.e., for $\varphi \in C_{0}^{\infty}\left(B_{\rho}\right)$

$$
\left(\int_{B_{\rho}}|\varphi|^{4} d \mu_{0}\right)^{1 / 2} \leq A_{0}\left(\int_{B_{\rho}}\left|\nabla^{0} \varphi\right|^{2} d \mu_{0}\right) .
$$

Take $\varphi=e^{\lambda} f$, since $E_{g}(f)=E_{g_{0}}(\varphi)$

$$
\begin{aligned}
\left(\int|f|^{4} d \mu\right)^{1 / 2} & =\left(\int|\varphi|^{4} e^{-4 \lambda} d \mu\right)^{1 / 2}=\left(\int|\varphi|^{4} d \mu_{0}\right)^{1 / 2} \\
& \leq A_{0}\left(\int|\nabla \varphi|^{2} d \mu_{0}\right) \\
& \leq A_{0}\left[E_{g_{0}}(\varphi)-\frac{1}{6} \int R_{0} \varphi^{2} d \mu_{0}\right] \\
& \leq A_{0}\left[E_{g}(f)-\frac{1}{6} \int R_{0} \varphi^{2} d \mu_{0}\right] \\
& \leq A_{0}\left[\int|\nabla f|^{2} d \mu+\frac{1}{6} \int\left(R-R_{0} e^{-2 \lambda}\right) f^{2} d \mu\right]
\end{aligned}
$$

Let $\Omega=\left\{p \in B_{\rho}: R-R_{0} e^{-2 \lambda}>-K\right\}, K>0$, estimate

$$
\begin{aligned}
& \int_{B_{\rho}}\left(R-R_{0} e^{-2 \lambda}\right) f^{2} d \mu \\
& \quad=\int_{\Omega}\left(R-R_{0} e^{-2 \lambda}\right) f^{2} d \mu+\int_{B_{\rho}-\Omega}\left(R-R_{0} e^{-2 \lambda}\right) f^{2} d \mu \\
& \quad \leq \int_{\Omega}\left(R-R_{0} e^{-2 \lambda}\right) f^{2} d \mu
\end{aligned}
$$


Now consider $\Omega^{\prime}=\Omega \cap\{\lambda<0\}$, then over $\Omega^{\prime}$

$$
\begin{aligned}
-6 \Delta_{0}(-\lambda) & =R_{0}-e^{2 \lambda} R-6\left|{ }^{0} \lambda\right|^{2} \\
& \leq R_{0}-e^{2 \lambda} R \leq K e^{2 \lambda} \leq C+K(-\lambda) .
\end{aligned}
$$

That is, for $h=-\lambda>0$, we have

$$
-\Delta_{0} h \leq \frac{1}{6} K h+C,
$$

over $\Omega^{\prime}$. But from Lemma 3.2, one has

$$
\int_{\Omega^{\prime}} h^{2} d \mu_{0} \leq C
$$

and

$$
\int_{\Omega^{\prime}} K^{p} d \mu_{0} \leq C, \quad p>2=\frac{n}{2}, n=4 .
$$

Then Moser iteration as in [Ch1, Theorem 3.3] implies

$$
-\lambda \leq C
$$

over $\Omega^{\prime}$ and then

$$
\lambda \geq-C
$$

on $\Omega$.

Therefore, from (3.3),

$$
\int_{B_{\rho}}\left(R-R_{0} e^{-2 \lambda}\right) f^{2} d \mu \leq \int_{\Omega} R f^{2} d \mu+C \int_{B_{\rho}} f^{2} d \mu .
$$

This and (3.2) imply

$$
\begin{aligned}
& \left(\int_{B_{\rho}}|f|^{4} d \mu\right)^{1 / 2} \leq A_{0}\left[\int_{B_{\rho}}|\nabla f|^{2} d \mu+\int_{\Omega} R f^{2} d \mu+C \int_{B_{\rho}} f^{2} d \mu\right] \\
& \leq A_{0}\left[\int_{B_{\rho}}|\nabla f|^{2} d \mu+\left(\int_{B_{\rho}} R^{2} d \mu\right)^{1 / 2}\left(\int_{B_{\rho}} f^{4} d \mu\right)^{1 / 2}+C \int_{B_{\rho}} f^{2} d \mu\right] .
\end{aligned}
$$
then

If $\int_{B_{\rho}} R^{2} d \mu$ is sufficiently small such that $A_{0}\left(\int_{B_{\rho}} R^{2} d \mu\right)^{1 / 2} \leq 1 / 2$,

$$
\left(\int_{B_{\rho}} f^{4} d \mu\right)^{1 / 2} \leq C_{s}\left[\int_{B_{\rho}}|\nabla f|^{2} d \mu+\int_{B_{\rho}} f^{2} d \mu\right],
$$

for some constant $C_{s}=C\left(A_{0}\right)$. 
Now we are ready to have the $C^{0}$-bound of solution of (1.3).

(I) The upper bound estimate:

Since

$$
\Delta \lambda=e^{-2 \lambda}\left(\Delta_{0} \lambda+2|\stackrel{0}{\nabla} \lambda|^{2}\right)
$$

then

$$
\begin{aligned}
R & =e^{-2 \lambda} R_{0}-6 e^{-2 \lambda}\left(\Delta_{0} \lambda+|\stackrel{0}{\nabla} \lambda|^{2}\right) \\
& =e^{-2 \lambda} R_{0}-6\left(\Delta \lambda-e^{-2 \lambda}|\stackrel{0}{\nabla} \lambda|^{2}\right) .
\end{aligned}
$$

This implies

$$
\begin{aligned}
-\Delta e^{\lambda / 2} & =-e^{\lambda / 2}\left[\frac{1}{2} \Delta \lambda+\frac{1}{4}|\nabla \lambda|^{2}\right] \\
& =-e^{\lambda / 2}\left[\frac{1}{2}\left(\frac{1}{6} e^{-2 \lambda} R_{0}-\frac{1}{6} R\right)+e^{-2 \lambda}|\stackrel{0}{\nabla} \lambda|^{2}+\frac{1}{4}|\nabla \lambda|^{2}\right] \\
& \leq \frac{1}{12}\left[R-e^{-2 \lambda} R_{0}\right] e^{\lambda / 2}
\end{aligned}
$$

That is, for $g=e^{\lambda / 2}, b=\frac{1}{12}\left|R-e^{-2 \lambda} R_{0}\right|$, we have

$$
-\Delta g \leq b g
$$

and from Corollary 2.3

$$
\int g^{2} d \mu \leq C ; \quad \int b^{2} d \mu \leq C .
$$

Now combining Lemma 3.3, (3.4), (3.5) and Moser iteration as in [Ch1, Theorem 3.3], it follows

Proposition 3.4. There exists a constant $\kappa=\kappa\left(\kappa_{1} ; \int g^{2} d \mu ; \int b^{2} d \mu\right)$ such that if

$$
\int_{B_{\rho}\left(x_{0}\right)} R^{2} d \mu \leq \kappa,
$$

then, for any $0<\eta<1$, there is a constant $C=C(\rho, \kappa, \eta)$ such that

$$
\sup _{B_{(1-\eta) \rho}} e^{\lambda / 2} \leq C .
$$


Moreover, if we does not meet the condition as in (3.6). Thus, by using the blowing up argument at the point as in [Ch1, Lemma 5.2], we are able to estimate the supernorm of the solution of (1.3). More precisely, since $\int_{M} R^{2} d \mu \leq C$, for a fixed $t$, we have only finite point $\left\{v_{1}, \ldots, v_{m}\right\}$ such that the $L^{2}$-norm of scalar curvature over $B_{\rho}\left(v_{i}\right)$ are larger than $\kappa$. Fix $v=v_{i}$, take a neighborhood $N$ of $v$ such that $N \cap\left\{v_{1}, \ldots, v_{m}\right\}=\{v\}$. Let $r(p)=d(p, v)$ and assume that $B_{2 \rho_{0}} \subset N$ for some $\rho_{0}$. Now for each small $\rho$, define $\tilde{g}_{i j}=\frac{1}{\rho^{2}} g_{i j}=e^{2 \tilde{\lambda}} g_{i j}^{0}$. Fix $p_{0} \in N$, such that $r\left(p_{0}\right)=\rho<\rho_{0}$. For $n=4$, we have

$$
\int_{M} R^{2} d \mu=\int_{M} \widetilde{R}^{2} d \tilde{\mu}
$$

Then

$$
-\widetilde{\Delta} \tilde{g} \leq \tilde{b} \tilde{g} ; \quad \int_{M} \tilde{b}^{2} d \tilde{\mu} \leq C
$$

and

$$
\int_{\widetilde{B}_{1 / 2}\left(p_{0}\right)} \tilde{g}^{2} d \tilde{\mu}=\int_{B_{\rho / 2}\left(p_{0}\right)} e^{5 \tilde{\lambda}} d \mu_{0} \leq \rho^{-5} \int_{B_{\rho / 2}\left(p_{0}\right)} e^{5 \lambda} d \mu_{0},
$$

where $\tilde{g}=e^{\tilde{\lambda} / 2}$ and $\frac{1}{\rho^{2}} e^{2 \lambda}=e^{2 \tilde{\lambda}}$.

Since

$$
\int_{B_{\rho / 2}\left(p_{0}\right)} R^{2} d \mu=\int_{\widetilde{B}_{1 / 2}\left(p_{0}\right)} \widetilde{R}^{2} d \tilde{\mu}
$$

take $\rho$ sufficiently small, we have

$$
\int_{B_{\rho / 2}\left(p_{0}\right)} R^{2} d \mu \leq \kappa
$$

On the other hand, since we have the local Sobolev constant bound for $g_{i j}$ as in Lemma 3.3, it still holds for $\tilde{g}_{i j}([\mathrm{Ch} 1,(5.2)])$. Then again follows the Moser iteration

$$
\sup \tilde{g}=\sup e^{\tilde{\lambda} / 2} \leq C\left\|e^{\tilde{\lambda} / 2}\right\|_{L_{2}} \leq C \rho^{-5 / 2}\left(\int_{B_{\rho / 2}\left(p_{0}\right)} e^{5 \lambda} d \mu_{0}\right)^{1 / 2}
$$

on $\widetilde{B}_{1 / 2}\left(p_{0}\right)$.

LEMMA 3.5. Under the flow (1.3), there exists a constant $C$ such that

$$
\int_{B_{\rho}(x)} e^{4 \lambda} d \mu_{0} \leq C \rho^{4} .
$$


We will proof the Lemma in the end of (I).

Now from (3.7) and (3.8), for $x \in \widetilde{B}_{1 / 2}\left(p_{0}\right), \widetilde{B}_{\rho}(x) \subset \widetilde{B}_{1 / 2}\left(p_{0}\right)$,

$$
\begin{aligned}
\int_{\widetilde{B}_{\rho}(x)} \tilde{g}^{2} d \tilde{\mu} & \leq C \rho^{-5}\left(\int_{B_{\rho / 2}\left(p_{0}\right)} e^{5 \lambda} d \mu_{0}\right) \int_{\widetilde{B}_{\rho}(x)} d \tilde{\mu} \\
& \leq C \rho^{-5} \int_{B_{\rho^{2}}(x)} e^{4 \tilde{\lambda}} d \mu_{0} \leq C \rho^{-9} \int_{B_{\rho^{2}}(x)} e^{4 \lambda} d \mu_{0} \leq C \rho^{-1} .
\end{aligned}
$$

Then

$$
\sup e^{\tilde{\lambda} / 2} \leq C\left\|e^{\tilde{\lambda} / 2}\right\|_{L_{2}} \leq C \rho^{-1 / 2}
$$

But

$$
e^{2 \tilde{\lambda}}=\rho^{-2} e^{2 \lambda}
$$

It follows

$$
e^{2 \lambda} \leq C
$$

on $B_{\rho / 2}\left(p_{0}\right)$ and as $r(p) \rightarrow 0$, we get $\lambda \leq C$ on $B_{r_{0}}(v)$ for small $r_{0}$.

All these imply

$$
\lambda \leq C
$$

on $M$.

Proof of (3.8). As before, we have

$$
-\Delta_{0} e^{\lambda}=\frac{1}{6}\left[e^{2 \lambda} R-R_{0}\right] e^{\lambda}
$$

In case of $R<0$, it follows

$$
-\Delta_{0} e^{\lambda} \leq \frac{1}{6} R_{0} e^{\lambda}
$$

Again from Moser iteration with respect to $g_{0}$, one has

$$
\lambda \leq C
$$

for $R<0$.

Then, for $B_{\rho}^{-}=B_{\rho} \cap\{R<0\}$

$$
\int_{B_{\rho}^{-}} e^{4 \lambda} d \mu_{0} \leq C \int_{B_{\rho}} d \mu_{0} \leq C \rho^{4} .
$$


On the other hand, for $B_{\rho}^{+}=B_{\rho} \cap\{R \geq 0\}$, the same argument as in [W, Proposition 5.4], the exterior unit normal vector $\nu_{+}$of $\partial B_{\rho}^{+}$has $D_{\nu_{+}} R \leq 0$,

$$
\begin{aligned}
\frac{d}{d t} \int_{B_{\rho}^{+}} e^{4 \lambda} d \mu_{0} & =4 \int_{B_{\rho}^{+}} e^{4 \lambda} \Delta R d \mu_{0}=4 \int_{B_{\rho}^{+}} \Delta R d \mu \\
& =4 \int_{\partial B_{\rho}^{+}} D_{\nu_{+}} R d \sigma \leq 0 .
\end{aligned}
$$

It follows

$$
\int_{B_{\rho}^{+}} e^{4 \lambda} d \mu_{0} \leq \int_{B_{\rho}} e^{4 \lambda_{0}} d \mu_{0} \leq C \rho^{4}
$$

This completes the proof of (3.8).

(II) The lower bound estimate:

The same method as in the previous (I), firstly, for $h=e^{-\lambda}, d=$ $\frac{1}{6}\left|R-e^{-2 \lambda} R_{0}\right|$, we have

$$
-\Delta h \leq d h
$$

and

$$
\int h^{2} d \mu \leq C ; \quad \int d^{2} d \mu \leq C .
$$

Then, again Moser iteration and the blowing up argument, the lower bound

$$
e^{-\lambda} \leq C
$$

is followed easily.

Then we have the $C^{0}$-bound of solution of (1.3):

THEOREM 3.6. Under the flow (1.3), there exists a constant $C=$ $C\left(\int e^{5 \lambda} d \mu_{0}, \lambda_{0}, g_{0}\right)$, such that

$$
\|\lambda\|_{L^{\infty}(M)} \leq C,
$$

for $t \in[0, T)$. Moreover, we have

$$
\|\lambda(t)\|_{W_{1,4}} \leq C .
$$

for $t \in[0, T)$. 
Proof. Since

$$
\int_{M}|R|^{2} d \mu \leq C
$$

then

$$
\int_{M} e^{-2 \lambda}\left(\Delta_{0} e^{\lambda}\right)^{2} d \mu_{0} \leq C
$$

But $\|\lambda\|_{L_{\infty}} \leq C$, it follows

$$
\int_{M}\left(\Delta_{0} e^{\lambda}\right)^{2} d \mu_{0} \leq C
$$

This implies

$$
\left\|e^{\lambda}\right\|_{W_{2,2}} \leq C
$$

and from Sobolev imbedding theorem $W_{2,2} \subset W_{1,4}$ for $n=4$, we have

$$
\|\lambda\|_{W_{1,4}} \leq C
$$

For higher order estimates, it is straightforward, we refer to [Chru] and $[\mathrm{CW}]$ for details.

TheOrem 3.7. ([Chru, Proposition 4.1], [CW]) The same assumptions as in the previous lemma. There exists a constant $C=C\left(\left\|\lambda_{0}\right\|_{W_{2,2}}, g_{0}, T\right)$, $l \geq 2$ such that

$$
\left\|\nabla^{0} \lambda(p, t)\right\|_{L_{2}} \leq C
$$

for $t \in[0, T)$.

Then the first part of main Theorem will follow easily from Theorem 3.6 and Theorem 3.7.

\section{$\S 4$. Asymptotic convergence of solutions of the Calabi flow on Einstein 4-manifolds}

In the previous sections, we show the following bound

$$
\int e^{5 \lambda} d \mu_{0} \leq\left(C_{2}+C_{3} t\right)
$$

and the $C^{0}$-bound

$$
\sup _{p \in M_{t}}|\lambda(p, t)| \leq C(T), \quad 0 \leq t<T
$$


Then we have the long time existence of solution of (1.3). However, at the previous steps, the $C(T)$ as in (4.1) may blow up as $t \rightarrow \infty$, but if the background metric $g_{0}$ is Einstein, then

$$
\int e^{5 \lambda} d \mu_{0} \leq C_{1}
$$

It follows we have the uniformly bound on $C(T)$ and $\|\lambda\|_{W_{k, 2}}$.

In this section, we will show that there exists a subsequence of solutions of (1.3) converges to a constant scalar curvature metric ([CW]).

THEOREM 4.1. Under the flow (1.3), if the background metric $g_{0}$ is Einstein. Then there exists a subsequence $\left\{t_{j}\right\}$ such that

$$
R \rightarrow R_{\infty}
$$

as $t_{j} \rightarrow \infty$ with

$$
\Delta R_{\infty}=0
$$

and

$$
g\left(t_{j}\right) \stackrel{C^{\infty}}{\longrightarrow} g_{\infty}
$$

Proof. Since

$$
-\frac{d}{d t} \int_{M} R^{2} d \mu=12 \int_{M}(\Delta R)^{2} d \mu
$$

then

$$
\int_{0}^{\infty} \int_{M}(\Delta R)^{2} d \mu d t<\infty
$$

and then there exists a subsequence $\left\{t_{j}\right\}$ such that

$$
\left.\int_{M}(\Delta R)^{2} d \mu\right|_{t_{j}} \rightarrow 0 \text { as } t_{j} \rightarrow \infty .
$$

Now since $\|\lambda\|_{W^{k, 2}} \leq C$ for all $0 \leq t_{j} \leq \infty$, we have

$$
\left.\int_{M}(\Delta R)^{2} d \mu_{0}\right|_{t_{j}} \rightarrow 0 \text { as } t_{j} \rightarrow \infty .
$$

Then elliptic estimates, interpolation inequalities yield

$$
R \stackrel{C^{\infty}}{\longrightarrow} R_{\infty}
$$


as $t_{j} \rightarrow \infty$ such that

$$
\Delta R_{\infty}=0
$$

and

$$
g\left(t_{j}\right) \stackrel{C^{\infty}}{\longrightarrow} g_{\infty}
$$

Then the second part of main Theorem follows easily.

Remark 4.1. From the uniqueness results of Einstein metrics in conformal class by M. Obata's results $([\mathrm{O}]), g_{\infty}$ is isometric to $g_{0}$.

\section{REFERENCES}

[A] T. Aubin, Nonlinear analysis on manifolds, Monge-Ampère Equations, Die Grundlehren der Math. Wissenschaften, Vol. 252, Springer-Verlag, New York, 1982.

[B] A. Besse, Einstein manifolds, Springer-Verlag, New York, 1986.

[C] C. B. Croke, Some isoperimetric inequalities and eigenvalue estimates, Ann. Sci. Ec. Norm. Super., 13 (1980), 419-435.

[Ca] E. Calabi, Extremal Kähler metrics, Seminars on Differential Geometry (S. T. Yau, ed.), Princeton Univ. Press and Univ. of Tokyo Press, Princeton, New York (1982), pp. 259-290.

[Ch1] S.-C. Chang, Critical Riemannian 4-manifolds, Math. Z., 214 (1993), 601-625.

[Ch2] Compactness theorems and the Calabi flow on Kaehler surfaces with stable tangent bundle, to appear.

[Ch3] Compactness theorems of extremal-Kähler manifolds with positive first Chern class, Annals of Global Analysis and Geometry, 17 (1999), 267-287.

[Ch4] The Calabi flow on Einstein manifolds, Lectures on Analysis and Geometry (S. T. Yau ed.), International Press, Hong Kong (1997), pp. 29-39.

[Ch5] The Calabi flow on surfaces, to appear.

[Chru] P. T. Chruściel, Semi-global existence and convergence of solutions of the Robinson-Trautman (2-dimensional Calabi) equation, Commun. Math. Phys., 137 (1991), 289-313.

[CW] S.-C. Chang and J. T. Wu, On the existence of extremal metrics for $L^{2}$-norm of scalar curvature on closed 3-manifolds, J. of Mathematics Kyoto University, 39-3 (1999), 435-454.

[Chav] I. Chavel, Eigenvalues in Riemannian geometry, Academic Press, New York, 1984.

[CY] S.-Y. A. Chang and P. Yang, Compactness of isospectral conformal metrics on 3-spheres, Comment. Math. Helvetici, 64 (1989), 363-374.

[O] M. Obata, The conjecture on conformal transformations of Riemannian manifolds, J. Diff. Geo., 6 (1971), 247-258. 
[S] R. Schoen, Conformal deformation of a Riemannian metric to constant scalar curvature, J. Diff. Geom., 20 (1984), 479-495.

[W] L.-F. Wu, The Ricci flow on complete $\mathbf{R}^{2}$, Communications in Analysis and Geometry, Vol 1, No. 3 (1993), 439-472.

Department of Mathematics

National Tsing Hua University

Hsinchu

Taiwan, 30043

R.O.C.

scchang@math.nthu.edu. tw 